\title{
Acolhimento como estratégia para alcançar a integralidade da assistência em hospital de média complexidade
}

\section{User embracement as a strategy to achieve integral assistance in hospitals of average complexity}

\author{
Carolina Brito Goulart ${ }^{1}$, Maria do Carmo Lourenço Haddad ${ }^{2}$, Marli Terezinha \\ Oliveira Vannuchi ${ }^{3}$, Mariana Angela Rossaneis ${ }^{4}$
}

\section{Resumo}

Acesso e acolhimento são elementos essenciais do atendimento, para que se possa atuar efetivamente sobre o estado de saúde do individuo. $\mathrm{O}$ acolhimento significa uma mudança na organização do processo de trabalho e uma nova diretriz para a instituição, além de uma postura diferenciada do profissional de saúde frente ao usuário. Este estudo descritivo foi realizado em um serviço de emergência de um hospital público de média complexidade, com o objetivo de identificar como o acolhimento com classificação de risco contribuiu para se alcançar à integralidade da assistência como um dos princípios do Sistema Único de Saúde. A coleta de dados foi realizada por meio da observação das estratégias utilizadas no acolhimento do usuário. A análise dos dados deu-se por meio do relato da realidade vivenciada no pronto socorro da instituição onde o estudo foi realizado, após a implantação do acolhimento com avaliação e classificação de risco, segundo a percepção dos autores. A implantação dessa estratégia de acolhimento foi um avanço para a instituição no reconhecimento da saúde como direito determinando a redução das filas de espera dos usuários, melhora no atendimento e satisfação do usuário e de funcionários da instituição, tornando o atendimento resolutivo, ético, integral e humanizado para os usuários que procuram o serviço.

Palavras-chaves: Acolhimento. Humanização da Assistência. Serviço Hospitalar de Emergência.

\begin{abstract}
Access and embracement are essential assistance elements in order to act effectively on an individual's health condition. Admission means a change in the work process organization and a new guideline for the institution, including a different attitude of the heath professional towards the user. The objective of this study was to describe on an experience carried out in an emergency service of an average complexity public hospital, to identify how an embracement with risk classification has contributed to the integrality of the assistance as one of the principles of the Single Health System. Data were collected through observation of the strategies used during the admission of users. Data analysis was carried out through reports on the reality experienced in the Emergency Room of the institution where
\end{abstract}

${ }^{1}$ Enfermeira. Pós Graduada em Gerência dos Serviços de Enfermagem na modalidade residência pela Universidade Estadual de Londrina, Londrina-PR. E-mail: carol.goulart@sercomtel.com.br

${ }^{2}$ Enfermeira. Doutora em Enfermagem Fundamental. Docente do Departamento de Enfermagem da Universidade Estadual de Londrina, Londrina-PR. E-mail: haddad@sercomtel.com.br

${ }^{3}$ Enfermeira. Doutora em Saúde Pública. Docente do Departamento de Enfermagem da Universidade Estadual de Londrina, Londrina-PR. E-mail: vannuchi@sercomtel.com.br

${ }^{4}$ Enfermeira. Mestranda do Programa de Pós-Graduação em Enfermagem da Escola de Enfermagem da Universidade Estadual de São Paulo de Ribeirão Preto, Ribeirão Preto-SP. E-mail: rossaneis@usp.br 
the study was done, after the implementation of embracement with evaluation and classification of risk, according to the authors' perceptions. The implementation of this admission strategy represented an advance for the institution which recognized health as a right, reduced users' waiting time (long lines) and improved the service and satisfaction of users and employees, turning the assistance into something ethical, humanized and solution-based for service users.

Key words: User Embracement. Humanization of Assistance. Emergency Service, Hospital.

\section{Introdução}

O Sistema Único de Saúde (SUS) é uma política organizacional para o reordenamento dos serviços e opções de saúde estabelecida pela constituição de 1988. A lei 8.080 de 1990 caracteriza o SUS como sendo um conjunto de ações e serviços, prestados por órgãos e instituições publicas, com o objetivo de reunir todas as instituições sejam elas publicas e privadas em um único sistema de saúde, além de oferecer uma atenção integral à saúde (FONTOURA, MAYER, 2006).

A integralidade é um dos princípios constitucionais do SUS que garante ao usuário o direito de acesso a todas as esferas de atenção em saúde desde atos preventivos, curativos, individuais e coletivos (GOMES; PINHEIRO 2005).

Para se alcançar a integralidade das ações é preciso trabalhar com um conceito mais amplo de saúde como trabalho em equipe, cuidado ao paciente e suas necessidades buscando uma assistência maior, transformadora, centrada no individuo, envolvendo a valorização do cuidado e o acolhimento (FONTOURA, MAYER, 2006).

$\mathrm{O}$ acolhimento enquanto uma das estratégias da política nacional de humanização (PNH) propõe inverter a lógica da organização e do funcionamento das instituições de saúde para que o serviço seja organizado de forma a atender melhor o usuário, e se modifique o modelo assistencial, que antes era centrado no médico e atualmente deve ser centrado em uma equipe multiprofissional ou equipe de acolhimento, empenhada em escutar o usuário, comprometendo-se em solucionar seu problema de saúde (BRASIL, 2006).
Desta forma, o presente trabalho tem como objetivo relatar como o acolhimento com classificação de risco contribuiu para alcançar à integralidade da assistência como um dos princípios do Sistema Único de Saúde em serviço de emergência de um hospital público de média complexidade.

\section{Material e métodos}

Neste estudo descritivo buscou-se descrever como o acolhimento com avaliação e classificação de risco, implantado em um hospital público de média complexidade, favoreceu a construção de uma relação de compromisso entre usuários e equipe de saúde.

O estudo foi realizado em um hospital público de média complexidade localizado na região norte do Estado do Paraná, Brasil, que presta atendimento ao SUS e possui 56 leitos distribuídos entre unidades de clínica médica, clínica cirúrgica, pediatria e pronto-socorro.

O pronto-socorro funciona 24 horas diárias e atente os pacientes encaminhados pelo Serviço de Atendimento Móvel de Urgência (SAMU), Serviço Integrado de Atendimento ao Trauma em Emergência (SIATE), Unidades Básicas de Saúde (UBSs) e demanda espontânea. Em média são realizados 6.000 atendimentos e 300 internações por mês. O centro cirúrgico realiza cirurgias eletivas de pequeno e médio porte.

A instituição conta com 220 funcionários entre profissionais terceirizados e trabalhadores de saúde 
contratados pelo estado

Os dados foram coletados durante o estágio da residência de Gerência dos serviços de enfermagem da Universidade Estadual de Londrina-Pr na instituição em estudo, entre os meses de fevereiro a agosto de 2008. Durante este período foram realizadas reuniões semanais entre a enfermeira do serviço, uma residente e duas docentes para analisar e discutir as práticas de atendimento ao usuário utilizando a estratégia do acolhimento com avaliação e classificação de risco na instituição em estudo. As discussões foram fundamentadas na metodologia problematizadora, a qual enseja, a partir da observação da realidade existente, desenvolver o pensamento crítico de modo a favorecer sua transformação (TEIXEIRA et al., 2009).

A análise dos dados deu-se por meio do relato da realidade vivenciada no pronto socorro da instituição de estudo, após a implantação do acolhimento com avaliação e classificação de risco relacionando com a bibliografia encontrada sobre o tema.

Para a realização do estudo obteve-se a autorização da Direção Geral da instituição.

\section{Resultados e discussão}

Sistema único de saúde, integralidade e acolhimento

A partir do descontentamento e insatisfação em relação ao atendimento a saúde no Brasil nas décadas de 70 e 80 , foi criado o SUS. A constituição federal de 1988 determinou em seu artigo 196 que saúde é um dever do estado e direito de todos, sendo o SUS um sistema público, organizado e orientado no sentido do interesse público (PINHO et al, 2007).

As leis 8080/90 e 8142/90, chamadas Leis Orgânicas da Saúde (LOS), são leis complementares que detalham a organização e o funcionamento do SUS estabelecido pela constituição federal. A Lei 8080/90 dispõe sobre as condições para a promoção, proteção e recuperação da saúde, a organização e o funcionamento dos serviços correspondentes e a Lei 8142/90 dispõe sobre a participação da comunidade na gestão do SUS e sobre as transferências intergovernamentais de recursos financeiros na área da saúde (PALHA; VILLA, 2003).

A integralidade, como um dos princípios do SUS exige novos padrões de relacionamento entre serviços e o usuário. Esta voltada para várias concepções e sentidos, porém, seu objetivo está na qualidade do atendimento prestado ao usuário, envolvendo questões como cuidado e acolhimento (FONTOURA, MAYER, 2006).

A partir disso, podemos reconhecer nas estratégias de melhoria de acesso o desenvolvimento de práticas integrais, o acolhimento. $\mathrm{O}$ acolhimento propõe inverter a lógica da organização e do funcionamento dos serviços de saúde (GOMES; PINHEIRO 2005).

Acolhimento consiste na humanização das relações entre trabalhadores e o serviço de saúde. A afinidade entre os dois resulta em uma relação de escuta e responsabilização para que se estabeleça um processo de trabalho centrado no interesse dos usuários (RAMOS; LIMA, 2003).

O acolhimento significa humanização, significa reconhecer os sujeitos como pessoas que tem desejos, necessidades e direitos. Dessa forma, a incorporação da proposta de acolhimento pode contribuir para uma efetiva responsabilização clinica e sanitária por parte do sistema de saúde, além de construir vínculos entre usuários e trabalhadores. Isso implica em incorporar esta diretriz na construção das políticas de saúde construindo e fortalecendo o SUS (TAKEMOTO; SILVA, 2007).

$\mathrm{O}$ acolhimento consiste na abertura dos serviços para a demanda e responsabilização por todos os problemas de saúde de uma determinada população. O serviço deve adaptar técnicas e combinar atividades, adequar recursos escassos e sociais, culturais e econômicos passados, pois a população procura instituições resolutivas, receptivos e dentro das proximidades de casa (SCHIMITH; LIMA, 
2004).

Quando o usuário procura uma instituição, geralmente, ele deseja ajuda e a equipe que esta a sua espera deve estar preparada de maneira adequada para se relacionar e atender esse usuário de forma clara, tranquila, agradável e resolutiva (CAMELO et al., 2000) .

Para uma instituição de saúde ou qualquer outra alcançar o acolhimento é preciso que haja relações respeitosas entre todos os membros da equipe. Os funcionários que não vivenciam em seu cotidiano relações respeitosas de amizade e comprometimento, não acolheram seus pacientes além de sentir-se descomprometidos com o atendimento que irão prestar (ROSSI; LIMA, 2005)

Acolhimento e classificação de risco no cotidiano do pronto socorro

Acesso e acolhimento são elementos essenciais do atendimento, para que se possa atuar efetivamente sobre o estado de saúde do individuo. Nos serviços públicos são observados vários problemas em relação ao acesso e acolhimento. Em relação à área física, geralmente sempre desaconchegantes e pequenas, sem local de espera, filas, qualificação inadequada dos profissionais, falta de materiais e funcionários e o relacionamento frio e desumano em algumas instituições (RAMOS; LIMA, 2003).

A postura dos trabalhadores reflete no acolhimento e atendimento aos usuários. A qualidade do atendimento é marcada por boa recepção pela equipe, respeito ao usuário, limpeza e organização, pontualidade no atendimento, informações concretas e precisas, bom desempenho profissional, relacionamento humanizado, interesse e preocupação com o usuário. Além da influência da opinião de outros usuários sobre a instituição e os serviços (TAKEMOTO; SILVA, 2007).

A baixa resolutividade e qualidade das instituições de saúde, atendimento precário, falta de políticas públicas, crenças da população, estrutura organizacional das instituições levam o usuário a procuram atendimento onde seja mais rápido $\mathrm{e}$ resolutivo (FONTOURA; MAYER, 2006).

A unidade de emergência é parte integrante na estrutura da instituição. Como a maioria dos hospitais públicos brasileiros, possui problemas no atendimento aos usuários que procuram o pronto socorro, devido à área física inadequada $\mathrm{e}$ o numero reduzido de profissionais, ocasionando em superlotação, transtornos para usuários e funcionários e lentidão no atendimento (MARQUES; LIMA, 2007).

A maioria da população para driblar uma longa fila de espera por consulta de exames nas UBSs procura o pronto socorro do hospital como possibilidade de ser atendido sobrecarregando as unidades de pronto socorro. Dessa forma o atendimento ao usuário fica comprometido, gerando descontentamento e insatisfação aos que procuram por este serviço. Grande parte da população procura o pronto socorro não só em casos agudos, mas também para complementar o atendimento já recebido na unidade básica de saúde ${ }^{(13)}$.

Para atender as propostas da Política Humanização do Ministério da Saúde, o hospital onde o estudo foi realizado, também preocupado com a qualidade na assistência, satisfação do usuário e saúde do trabalhador atua com propostas para a efetivação do acolhimento com classificação de risco na unidade do pronto socorro.

A classificação de risco é um processo de identificação de pacientes que necessitam de atendimento imediato de acordo com o risco e grau de sofrimento. A classificação é feita conforme sua necessidade especifica. A cor vermelha indica emergência, cor amarela urgência, cor verde prioridade não urgente e cor azul indica consulta de prioridade não urgente.

Os pacientes são atendidos por ordem de chegada pelo profissional de registro, onde é realizada a ficha de atendimento. Após a confecção da ficha, o paciente aguarda a chamada pelo auxiliar de enfermagem na recepção do hospital 
que fica do lado de fora da instituição. $\mathrm{O}$ auxiliar de enfermagem, dentro do hospital, faz o acolhimento com classificação de risco chamando os pacientes para a consulta com o clínico geral conforme sua classificação. Na recepção, não há uma sala privativa para realizar a avaliação de risco, nem mesmo sala de espera dentro da instituição e também não há sinalização e identificação de fluxo do paciente na instituição.

Com a sensibilização dos gestores, profissionais de saúde e funcionários do hospital, foi realizada a divulgação da proposta para todo o quadro de funcionários, treinamentos sobre acolhimento com classificação de risco para a equipe de enfermagem e também para os funcionários administrativos do pronto socorro, bem como divulgação das propostas para os usuários em forma de banner espalhados na entrada da instituição e pronto-socorro. Além de um fluxograma de atendimento, oficinas e discussão e elaboração de protocolos para direcionar o atendimento.

Após a implantação do acolhimento com avaliação e classificação de risco ficou ainda mais evidente a demanda reprimida, não apenas para os serviços de atenção primária, como também para os que aguardam procedimentos especializados para diagnósticos de patologias ou cirurgias e buscam os prontos-socorros para tratamento dos sintomas.

Apesar da estrutura física deficiente para receber o paciente adequadamente, o acolhimento com avaliação e classificação de risco configurouse uma intervenção decisiva na reorganização do fluxograma de atendimento no serviço de emergência da instituição onde o estudo foi realizado, reordenando o processo de trabalho de forma a atender os diferentes graus de necessidades ou sofrimento de cada indivíduo com maior agilidade e resolutividade.

\section{Considerações finais}

O acolhimento significa uma mudança na organização do processo de trabalho e uma nova diretriz para a instituição, além de uma postura diferenciada do profissional de saúde frente ao usuário. Com isso, o resultado esperado é a redução das filas de espera dos usuários, melhora no atendimento e satisfação do usuário.

Para que a consolidação do acolhimento seja alcançada os colaboradores devem ser atendidos em suas necessidades de supervisão, apoio institucional e educação permanente para que possam refletir e se conscientizar que a atenção à saúde deve ser humanizada, integral e considerada como prioridade.

A implantação do acolhimento com classificação de risco foi um avanço para a instituição no reconhecimento da saúde como direito. Para que o atendimento seja mais resolutivo, ético, integral e humanizado para os usuários que procuram atendimento no serviço. Caracterizando assim, uma fase de transição para melhorias a saúde pública.

È sabido que muitas ações e melhorias precisam e irão ser realizadas na instituição, entretanto houve mudanças significativas para se tentar alcançar a humanização através do acolhimento com classificação de risco.

\section{Referências}

BRASIL. Ministério da Saúde. Secretária de Atenção a Saúde. Núcleo Técnico da Política Nacional de Humanização. Acolhimento nas práticas de produção de saúde. 2ed. Brasília: Ministério da Saúde: 2006.

CAMELO, S.H.H.; ANGERAMI, E.L.S.; SILVA, E.M.; MISHIMA, S.M. Acolhimento à clientela: estudo em unidades básicas de saúde no município de Ribeirão Preto. Revista eletrônica de enfermagem. Goiânia, v.8, n.4, p.30-37, ago. 2000. 
GOMES, M.C.P.A.; PINHEIRO, R. Acolhimento e vínculo: práticas de integralidade na gestão do cuidado em saúde em grandes centros urbanos. Interface - Comunicação, Saúde e Educação. Botucatu, v.9, n.17, p.287-301, mai/ago.; 9(17). 2005

FONTOURA, R.T., MAYER, C.N. Uma breve reflexão sobre a integralidade. Revista Brasileira de enfermagem. Brasília, v.59, n.4, p.532-572, jul. 2006.

MARQUES, G.Q.; LIMA, M.A.D.S. Demandas de usuários a um serviço de pronto atendimento e seu acolhimento ao sistema de saúde. Revista latino-americana de enfermagem. Ribeirão Preto, v.15, n.1, p.13-19, jan/fev. 2007.

MIRANDA, J.M.; ASSUNÇÃO, M.A.F.; NOGUEIRA, R.Y.S. Práticas de humanização: acolhimento com avaliação e classificação de risco no pronto socorro do Hospital Zona Sul de Londrina. 2007. [monografia]. p.78. Londrina (PR): Universidade Estadual de Londrina; 2007.

PALHA, P.F.; VILLA, T.C.S. A descentralização como eixo norteador na reorganização e operacionalização dos princípios do Sistema Único de Saúde. Revista da Escola de Enfermagem da USP. Ribeirão Preto, v.37, n.3, p.19-26, set. 2003.

PINHO, L.B.; KANTORSKI, L.P.; SAEKI, T.; DUARTE, M.L.C.; SOUSA, J. A integralidade no cuidado em saúde: um resgate de parte da produção cientifica da área. Revista eletrônica de enfermagem. Goiânia, v.9, n.3, p.835-846, set/dez. 2007.

ROSSI, F.R.; LIMA, M.A.D.S. Acolhimento: tecnologia leve nos processos gerenciais do enfermeiro. Revista Brasileira de Enfermagem. Brasília, v.58, n.3, p.305-310, mai/jun. 2005.

RAMOS, D.D.; LIMA, M.A.D.S. Acesso e acolhimento aos usuários em uma unidade de saúde de Porto Alegre, Rio Grande do Sul, Brasil. Cadernos de Saúde Pública. Rio de Janeiro, v.19, n.1, p.27-34, jan/fev. 2003.
SCHIMITH, M.D.; LIMA, M.A.D.S. Acolhimento e vínculo em uma equipe do Programa Saúde da Família. Cadernos de Saúde Pública. Rio de Janeiro, v.20, n.6, p. 1487-1494, nov/dez. 2004.

TAKEMOTO, M.L.S.; SILVA, E.M. Acolhimento e transformações no processo de trabalho de enfermagem em unidades básicas de saúde de Campinas, São Paulo, Brasil. Cadernos de Saúde Pública. Rio de Janeiro, v.23, n.2, p.331-340, jan/fev. 2007.

TEIXEIRA, R.C.; LABRONICE， L.M.; MAFTUM, M.A., MANTOVANI, M.F. Marco de referência humanística ao cuidado de enfermagem em uma unidade de saúde: relato de experiência. Ciência, Cuidado e Saúde. Maringá, v.8, n.3, p.484490, jul/set. 2009.
Recebido em: 27 de agosto de 2012 Aceito em: 14 de novembro de 2012 